\title{
Novel strategies in imaging
}

\author{
Tillmann Loch
}

Published online: 23 August 2011

(C) Springer-Verlag 2011

Urology imaging varies around the world. A recent survey by the ESUI (European Society of Urologic Imaging; full member of the European Association of Urology (EAU)) compared the different imaging modalities in different countries of Europe. This survey revealed dramatic differences as well as similarities in the application of imaging by radiologists and urologists. However, imaging play a key role in the daily routine of academic as well as in private practice urology.

Many innovating imaging approaches have been developed in the field of urology. Ultrasound seems to be the most commonly used modality in urologic imaging. Therefore, this issue focuses on novel imaging strategies in order to encourage and motivate further development of existing or new methods.

For many years, the ESUI has tried to promote and inform on new and standard strategies in and around the field of urologic imaging. As in every field, expertise and experience are necessary to achieve quality imaging. Furthermore, the consequences of preoperative diagnostic imaging and the need to learn from correct and incorrect interpretation lead to consistent improvement of the diagnostic quality. The ability to translate morphologic changes and anatomic findings into pathologic processes enables us to find adequate therapeutic strategies for our patients.

One problem of studies on imaging is the fact that when prospective or multicenter studies have been finalized and published, many of the methods are outdated and or have been even replaced by newer versions or more advanced

\section{T. Loch ( $\square)$}

Department of Urology, Diakonissenkrankenhaus Flensburg,

University Teaching Hospital, Flensburg, Germany

e-mail: lochti@diako.de technologies. Therefore, it is of utmost importance to report on new concepts and developments early.

In many cases, small but consistent improvements of existing technologies lead to more significant clinical improvement than dramatic new developments. In addition, transfer of techniques from other specialities to urology sometimes develop new concepts and understanding. A comparison of pathologic imaging findings with neighboring fields like gynecology, surgery, nuclear medicine, and oncology sometimes often leads to a better understanding of the significance of these findings and possibly better concepts.

Today, many improvements combine existing imaging modalities with digital algorithms and hi-tech computer technology to enable more objective and reproducible results. Most modern imaging systems have great digital storage and processing capabilities. Network solutions and large databases with expert systems seem to be the next step in modern imaging strategies by being able to spread the experience and expertise to connected users.

This topic issue is to inform you about different approaches to novel strategies in imaging and give you a chance to compare your experience and expertise with the presented information.

In one of the studies in this issue Grabski et al. ask the question, "Can prostate cancer diagnostic imaging be improved by a computer-based analysis of the transrectal ultrasound signal (C-TRUS) system?" Until recently, C-TRUS existed only as a stand-alone device. Now, C-TRUS has been developed into a network-compatible module (C-TRUS-MS). This new technology allows users to transmit C-TRUS images from any internet platform for C-TRUSMS investigation. The results indicate that C-TRUS-MS "online" achieves similar results as the stand-alone system, independent from the user's imaging experience. Furthermore, 
C-TRUS-MS has been able to detect carcinomas in patients without prior biopsies in high numbers and with only six targeted biopsies.

Smeenge et al. describe the current status of contrastenhanced ultrasound (CEUS) of the prostate and discuss new quantification techniques that can improve the accuracy even further. Quantification might decrease the user dependency and open the door for use in a routine clinical environment. A new generation of targeted microbubbles is now under pre-clinical testing and showed avid binding to VEGFR-2, a receptor up-regulated in prostate cancer due to angiogenesis. The first publications regarding a targeted microbubble ready for human use will be discussed. Ultrasound-assisted drug delivery gives rise to a whole new set of therapeutic options, also for prostate cancer. A major break-through in the future can be expected from the clinical use of targeted microbubbles, both for drug delivery, for prostate cancer diagnosis and treatment.

Walz et al. explore the ability of real-time elastography to correctly identify the prostate cancer index lesion as a possible tool for focal therapy. In 32 patients, real-time elastography was performed the day before prostatectomy. During the examination, the location of the main lesion suspicious for prostate cancer was prospectively recorded. Moreover, the results of the randomized multicore biopsies were also used to predict the location of the index lesion. The preoperative elastography results, the biopsy results and a combined use of elastography and biopsy results were then compared with the pathological results in order to calculate the diagnostic values for correct index lesion identification. They conclude that real-time elastography alone did not allow to identify the prostate cancer index lesion with satisfactory reliability. But the combination of real-time elastography and data from 12 randomized core biopsies shows promising ability to correctly identify the prostate cancer index lesion.

Martino et al. report that defining the site of recurrent disease for localized prostate cancer early after definitive treatment is a critical issue as it may greatly influence the subsequent therapeutic strategy or patient management. A systematic review of the literature from January 1995 to January 2011 was performed by searching Medline. Electronic searches were limited to the English language, and the keywords prostate cancer, RadioTherapy [RT], High Intensity Focused Ultrasound [HIFU], CryoTherapy [CRIO], Transrectal ultrasound [TRUS], Magnetic resonance $[\mathrm{MRI}], \mathrm{PET} / \mathrm{TC}$, and prostate biopsy were used. They conclude that it is likely that imaging will be used extensively in the future to detect and localize prostate cancer local recurrences before salvage treatment.

Wieczorek et al. discuss the role of various ultrasound modalities in the diagnostics of female pelvic floor disorders (PFD). Ultrasonography is the most widely available imaging modality. Due to the technical progress, novel transducers and more sophisticated software, providing more information about pelvic anatomy, have been recently introduced on the market. Some features of these transducers, such as higher frequency and multiplanar imaging enable better visualization of pelvic floor organs. The knowledge about advantages and limitations, and technical and physical properties of modern US could provide an integrated approach to imaging of PFD. Technical modalities, the wide availability of US techniques, and a better understanding of modern ultrasound capabilities could improve our knowledge about PFD and allow better assessment in pre- and post-surgical management.

Liguori et al. comment on the "acute scrotum" as a common emergency department (ED) presentation which can be defined as any condition of the scrotum or intrascrotal contents requiring emergent medical or surgical intervention. Although rarely fatal, acute scrotal pathology can result in testicle infarction and necrosis, testicular atrophy, infertility, and significant morbidity. The evaluation of acute scrotal pain can be challenging for the clinician initially examining and triaging the patient. They conclude that a scrotal ultrasound should always be performed in the presence of acute scrotal pain. Moreover, urologists should be able to perform a scrotal ultrasound but, if imaging does not supply a clear diagnosis, surgical exploration is still mandatory.

Siracusano et al. report on continuous innovations and clinical research in contrast-enhanced ultrasound (CEUS) technology for diagnosis of nephro-urological pathologies. By using a microbubble contrast agent and contrast-specific imaging software, CEUS is able to depict the micro- and macro-circulation of the targeted organ, which in turn leads to improved diagnostic performance. In particular, CEUS seems to be promising in the diagnosis of kidney ischemia, in the characterization of complex cystic lesions, and in lesions with equivocal enhancement in CT. CEUS provides information on tissue perfusion and may play a role in kidney mass characterization similar to that of contrastenhanced CT and MRI. In this context, the characterization of cystic lesions is probably the most consolidated field of application of contrast agents on kidney ultrasound imaging. CEUS seems to provide a good alternative to CT, especially in patients with contraindications to iodinated contrast agents.

Pilatz et al. investigate the diagnostic value of color Doppler ultrasound for defining the varicocele grade according to WHO criteria. They report on a total of 217 men (129 with clinical varicocele, 88 without clinical varicocele) who were investigated by physical examination and color Doppler ultrasound and categorized according to WHO varicocelecriteria (0, subclinical, I, II, and III). Diameter and reflux of the largest vein in the pampiniform 
plexus were measured bilaterally with the patient in the supine position in rest and during the Valsalva maneuver. To assess the possibility of differentiating varicocele grades by their venous diameters, optimal cut-point values were determined by receiver-operator characteristic (ROC) analysis. Their findings support the hypothesis that clinical varicoceles can be predicted with high accuracy based only on the diameter of testicular veins using cut-point values of $>2.45 \mathrm{~mm}$ in rest or $>2.95 \mathrm{~mm}$ during the Valsalva maneuver in supine position.

Wieczorek et al. describe from a radiologist point of view a quantitative assessment of urethral vascularity in nulliparous females using high-frequency endovaginal ultrasonography. They assess the vascular parameters in the urethra in these patients and compare the vascularity among various parts of the urethra, using high-frequency endovaginal ultrasonography (EVUS). They conclude that there is an excellent interobserver and intraobserver reproducibility in the majority of defined parameters. Vascularity differs in different parts of the urethra. Pixel Flux is a valuable tool for providing reproducible quantitative analysis of vascular parameters in the urethra. The authors are currently correlating vascularity perfusion, urodynamic studies, and ultrasound morphology of the urethra in various clinical pelvic floor disorders and hope to find parameters that might help in the management of urinary incontinence.

Schenck and Schneider introduce a standardized concept to check anastomosis, by evaluating urinary extravasation and by using a dTRUS. From 1999 to 2008, 1,479 radical prostatectomies were performed. Seven hundred and fiftytwo could be evaluated using the standardized concept of checking the anastomosis for complete tightness. The first 250 patients were evaluated by cystogram and dTRUS, the following 502 patients only by dTRUS postoperatively. The date of checking the anastomosis was determined by the urine color. They conclude that the urine color is essential to assess the risk of a persisting leakage at the anastomosis following radical prostatectomy. The method of dTRUS can replace the cystogram in checking for complete healing of the anastomosis, thus decreasing the exposure of the patient to X-rays of about $60 \mathrm{cGy} / \mathrm{cmÇ}$.

Loch discusses the role of innovative imaging-guided biopsies in patients with a history of negative biopsies but with a remaining suspicion of prostate cancer. According to international guidelines, a primary set of TRUS-guided systematic biopsy should consist of 10-12 tissue samples. If clinical suspicion of a prostate carcinoma persists, a secondary biopsy session should also involve 10-12 samples. The available innovative imaging techniques range from MRI, Doppler techniques with and without contrast agents, and a renaissance of elastography to computer-assisted evaluation of the TRUS signal information. All of these methods attempt to make more specific statements on the imaged tissue. Before routine clinical use, a review of the literature is recommended to be able to differentiate between the different methods. MRI techniques are highly sophisticated methods of high-resolution prostate imaging; guided biopsies, however, are not routine practice and not recommended in urological guidelines yet. In summary, if no carcinoma is found in a series of systematic biopsies, the author sees this as the ideal indication for innovative imaging techniques that might lead to specific detection. 